\title{
PRINCIPAL MEAN CURVATURE FOLIATIONS ON SURFACES IMMERSED IN $\mathbb{R}^{4}$
}

\author{
R. GARCIA, L. F. MELLO AND J. SOTOMAYOR
}

\begin{abstract}
Here are studied qualitative properties of the families of curves -foliations- on a surface immersed in $\mathbb{R}^{4}$, along which it bends extremally in the direction of the mean normal curvature vector. Typical singularities and cycles are described, which provide sufficient conditions, likely to be also necessary, for the structural stability of the configuration of such foliations and their singularities, under small $C^{3}$ perturbations of the immersion. The conditions are expressed in terms of Darbouxian type of the normal and umbilic singularities, the hyperbolicity of cycles, and the asymptotic behavior of singularity separatrices and other typical curves of the foliations. They extend those given by Gutierrez and Sotomayor in 1982 for principal foliations and umbilic points of surfaces immersed in $\mathbb{R}^{3}$. Expressions for the Darbouxian conditions and for the hyperbolicity, calculable in terms of the derivatives of the immersion at singularities and cycles, are provided. The connection of the present extension from $\mathbb{R}^{3}$ to $\mathbb{R}^{4}$ to other pertinent ones as well as some problems left open in this paper are proposed at the end.
\end{abstract}

\section{Position of the Problem, Results and Examples}

Let $\mathbb{M}^{2}$ be a 2-dimensional, compact, oriented, smooth manifold. Denote by $\mathcal{I}^{r, s}$ the space of immersions $\alpha$ of class $C^{r}$ of $\mathbb{M}^{2}$ into $\mathbb{R}^{4}$, with the $C^{s}$ topology, $r \geq s$. $\mathbb{M}^{2}$ and $\mathbb{R}^{4}$ are endowed with a once for all fixed orientation.

The projections of the pullback, $\alpha^{*}\left(\mathbb{R}^{4}\right)$, of the tangent bundle of $\mathbb{R}^{4}$ onto the tangent, $\mathbb{T M}^{2}$, and normal, $\mathbb{N}_{\alpha}$, bundles of an immersion $\alpha$ will be denoted respectively by $\Pi_{\alpha, t}$ and $\Pi_{\alpha, n}$. These vector bundles are endowed with the standard metrics induced by the Euclidean one, $<,>$, in $\mathbb{R}^{4}$.

Denote by $H_{\alpha}$ the normal mean curvature vector field of $\alpha$, which is defined by centers of the field of ellipses of normal curvature of $\alpha$. For any positive orthonormal tangent frame $\left\{e_{1}, e_{2}\right\}$, it holds that $H_{\alpha}=$

This work is supported CNPq Grant 476886/2001-5 and PRONEX/FINEP/MCT - Conv. 76.97.1080.00. 
$\Pi_{\alpha, n}\left(D e_{1}\left(e_{1}\right)+D e_{2}\left(e_{2}\right)\right) / 2$. See Little [10] and Garcia and Sotomayor 3.

The normal singularities of $\alpha$, defined by the zeros of $H_{\alpha}$, will be denoted by $\mathcal{S}_{n, \alpha}$. For generic immersions, when non-empty, $\mathcal{S}_{n, \alpha}$ is a discrete set of points at which $H_{\alpha}$ is transversal to the zero section of $\mathbb{N}_{\alpha}$. See Little [10] and Mello 11.

The unit normal mean curvature vector field of $\alpha, N_{\alpha}=\frac{H_{\alpha}}{\left|H_{\alpha}\right|}$, is defined on the complement of $\mathcal{S}_{n, \alpha}$. The unit vector field $B_{\alpha}=e_{1} \wedge e_{2} \wedge$ $N_{\alpha}$ is well defined for any positive orthonormal tangent frame $\left\{e_{1}, e_{2}\right\}$ and will be called the mean bi-normal vector field of $\alpha$.

The eigenvalues $k_{\alpha} \leq K_{\alpha}$ of the Weingarten operator $\mathcal{W}_{\alpha}=-\Pi_{\alpha, t} D N_{\alpha}$ of $\mathbb{T M}^{2}$ are called the principal mean curvatures of $\alpha$. The set of points $\mathcal{S}_{u, \alpha}$, where $k_{\alpha}=K_{\alpha}$, will be called the umbilic singularities of $\alpha$. Outside $\mathcal{S}_{\alpha}=\mathcal{S}_{n, \alpha} \cup \mathcal{S}_{u, \alpha}$, the singular set of $\alpha$, are defined the minimal, $L_{m, \alpha}$, and the maximal, $L_{M, \alpha}$, principal mean line fields of $\alpha$, which are the eigenspaces of $\mathcal{W}_{\alpha}$ associated respectively to $k_{\alpha}$ and $K_{\alpha}$. The integral foliations of these line fields, which are of class $C^{r-2}$ on the complement of $\mathcal{S}_{\alpha}$, will be denoted by $\mathcal{F}_{m, \alpha}$ and $\mathcal{F}_{M, \alpha}$.

In a local chart $(u, v)$ the principal mean curvatures lines of $\alpha$ are characterized as the solutions of the following quadratic differential equation:

$$
\left(F g_{H}-f_{H} G\right) d v^{2}+\left(E g_{H}-e_{H} G\right) d u d v+\left(E f_{H}-F e_{H}\right) d u^{2}=0,
$$

where $E=<\alpha_{u}, \alpha_{u}>, F=<\alpha_{u}, \alpha_{v}>, G=<\alpha_{v}, \alpha_{v}>$ are the coefficients of the first fundamental form $I_{\alpha}=\alpha^{*}<,>$ and $e_{H}=<$ $\alpha_{u u}, H_{\alpha}>, \quad f_{H}=<\alpha_{u v}, H_{\alpha}>, g_{H}=<\alpha_{v v}, H_{\alpha}>$ the coefficients of the second fundamental form relative to $N_{\alpha}$, denoted $I I_{N_{\alpha}}$, which have been multiplied by $\left|H_{\alpha}\right|$ to remove the denominators.

The left hand member of equation (II) is equivalent to the Jacobian, $J_{\alpha}$, of the quadratic forms $I_{\alpha}$ and $I I_{N_{\alpha}}$.

The principal mean configuration of $\alpha$ is defined by the quadruple $\mathcal{P}_{\alpha}=\left\{\mathcal{S}_{n, \alpha}, \mathcal{S}_{u, \alpha}, \mathcal{F}_{m, \alpha}, \mathcal{F}_{M, \alpha}\right\}$.

An immersion $\alpha$ in $\mathcal{I}^{r, s}$ is said to be $(r, s)$ - principal mean curvature structurally stable if it has a neighborhood $\mathcal{V}$ such that for every $\beta$ in $\mathcal{V}$ there is a homeomorphism $h_{\beta}$ mapping $\mathcal{S}_{n, \beta}$ and $\mathcal{S}_{u, \beta}$ respectively onto $\mathcal{S}_{n, \alpha}$ and $\mathcal{S}_{u, \alpha}$ and mapping the lines of the foliations $\mathcal{F}_{m, \beta}$ and $\mathcal{F}_{M, \beta}$ respectively onto those of the foliations $\mathcal{F}_{m, \alpha}$ and $\mathcal{F}_{M, \alpha}$. Denote by $\mathcal{E}^{r, s}$ the class of $(r, s)$ - principal mean curvature structurally stable immersions.

This global notion can be localized at the singularities: $\left\{\mathcal{S}_{n, \alpha}, \mathcal{S}_{u, \alpha}\right\}$ and at other invariant sets of the foliations, such as some of the principal mean curvature cycles, which are the periodic leaves of the foliations. 
A singularity of $\alpha$ is called Darbouxian if a) at all points of the projective line over it, $d J \neq 0$ and b) the Lie-Cartan Vector Field, given locally by

$$
X_{\alpha}=\left(J_{p}, p J_{p},-\left(J_{u}+p J_{v}\right)\right),
$$

with $J=J_{\alpha}$ defined in equation 1, is such that along the projective line over the singularity has only hyperbolic equilibria [7]. See Section 2 for more precise definition.

As in the standard $\mathbb{R}^{3}$ case, there are three Darbouxian types. In the case $D_{1}$ there is only one hyperbolic saddle. In the case $D_{2}$ there are three hyperbolic singular points, one node and two saddles. In the case $D_{3}$ there are three hyperbolic saddle points. The subscript $i$ in $D_{i}$ denotes the number of separatrices reaching the singularity. Conditions on the third order jet of $\alpha$ at a singularity to be Darbouxian and which discriminate its $D_{i}$ type will be given in Section 2, See Fig. 1 .

A principal mean curvature cycle $c$ of $\mathcal{F}_{m, \alpha}$ or $\mathcal{F}_{M, \alpha}$, is called $h y$ perbolic if the derivative of its first return - also called holonomy or Poincaré map, $\pi_{c}$, is different from 1. An integral expression for this derivative in terms of geometric curvature function along $c$ is given in Section 3 .

If the limit set of a leaf of a principal mean foliation is contained in the set of singular points and cycles it is said to be limit set trivial.

The main result of this paper can be stated now. The proof is outlined in Section 4 .

Theorem 1. Denote by $\Sigma^{(r, s)}$ the class of immersions which satisfy the conditions on Darbouxian singularities, hyperbolic cycles, nonconnection of singularity separatrices and triviality of the limit sets of all principal mean curvature lines. Then $\Sigma^{(r, s)}$ forms an open set in $\mathcal{I}^{r, s}$ and it is contained in $\mathcal{E}^{r, s}$ for $r \geq 4, s \geq 3$.

The analysis of the density of the class $\Sigma^{(r, s)}$ in $\mathcal{I}^{r, 2}$ will be postponed to a forthcoming paper. Meanwhile we will give some examples to confirm that $\Sigma^{(r, s)}$ is not empty.

Example 2. a) By composing with the stereographic projection of $\mathbb{R}^{3}$ into $\mathbb{S}^{3}$ the principal structurally immersions in $\mathbb{R}^{3}$ studied by Gutierrez and Sotomayor 5, are obtained elements in $\Sigma^{(r, s)}$. See 11.

b) Also, the subclass of Gutierrez and Sotomayor of immersions in $\mathbb{R}^{3}$ with non-vanishing mean curvature is contained inside $\Sigma^{(r, s)}$.

Theorem 1 partially extends a result of Gutierrez and Sotomayor [5] for the structural stability of principal configurations on surfaces in $\mathbb{R}^{3}$. 
For other pertinent extensions to immersions of surfaces into $\mathbb{R}^{4}$, see Section 5.

\section{Darbouxian Singularities}

In a Monge chart $(u, v)$, an immersion $\alpha$ is expressed as follows:

$$
\begin{aligned}
& \alpha(u, v)=\left(u, v, h_{1}(u, v), h_{2}(u, v)\right), \\
& h_{1}(u, v)=\frac{r_{1}}{2} u^{2}+s_{1} u v+\frac{t_{1}}{2} v^{2}+\frac{a_{1}}{6} u^{3}+\frac{d_{1}}{2} u^{2} v+\frac{b_{1}}{2} u v^{2}+\frac{c_{1}}{6} v^{3}+O(4), \\
& h_{2}(u, v)=\frac{r_{2}}{2} u^{2}+s_{2} u v+\frac{t_{2}}{2} v^{2}+\frac{a_{2}}{6} u^{3}+\frac{d_{2}}{2} u^{2} v+\frac{b_{2}}{2} u v^{2}+\frac{c_{2}}{6} v^{3}+O(4) .
\end{aligned}
$$

Let $B_{1}=\left(-\frac{\partial h_{1}}{\partial u},-\frac{\partial h_{1}}{\partial v}, 1,0\right), B_{2}=\alpha_{u} \wedge \alpha_{v} \wedge B_{1}$. Write $N_{i}=B_{i} /\left|B_{i}\right|, i=$ 1,2. Clearly $<\alpha_{u}, N_{i}>=<\alpha_{v}, N_{i}>=<N_{1}, N_{2}>=0$ and

$$
\begin{aligned}
& N_{1}(u, v)=\left(-r_{1} u-s_{1} v+O(2),-s_{1} u-t_{1} v+O(2), 1,0\right), \\
& N_{2}(u, v)=\left(-r_{2} u-s_{2} v+O(2),-s_{2} u-t_{2} v+O(2), O(2), 1+O(2)\right)
\end{aligned}
$$

The coefficients, $E, F, G$, of the first fundamental form, $I_{\alpha}$, induced by $\alpha$, and those of the second fundamental forms, $I I_{i \alpha}$, relative to $N_{i}$, denoted $e_{i}, f_{i}, g_{i}, i=1,2$, are calculated in the chart $(u, v)$ as follows:

$$
\begin{aligned}
& E(u, v)=1+O(2), \quad F(u, v)=O(2), \quad G(u, v)=1+O(2), \\
& e_{1}(u, v)=r_{1}+a_{1} u+d_{1} v+O(2), \quad e_{2}(u, v)=r_{2}+a_{2} u+d_{2} v+O(2), \\
& f_{1}(u, v)=s_{1}+d_{1} u+b_{1} v+O(2), \quad f_{2}(u, v)=s_{2}+d_{2} u+b_{2} v+O(2) \\
& g_{1}(u, v)=t_{1}+b_{1} u+c_{1} v+O(2), \quad g_{2}(u, v)=t_{2}+b_{2} u+c_{2} v+O(2)
\end{aligned}
$$

The components of $H=H_{\alpha}$ relative to a positive normal frame $N_{1}, N_{2}$ are given by $H_{1}=\frac{G e_{1}-2 F f_{1}+E g_{1}}{2\left(E G-F^{2}\right)}, H_{2}=\frac{G e_{2}-2 F f_{2}+E g_{2}}{2\left(E G-F^{2}\right)}$. With the coefficients from equations [5] in the chart $(u, v)$, we obtain

$$
\begin{aligned}
& H_{1}(u, v)=\frac{t_{1}+r_{1}}{2}+\frac{a_{1}+b_{1}}{2} u+\frac{c_{1}+d_{1}}{2} v+O(2) \\
& H_{2}(u, v)=\frac{t_{2}+r_{2}}{2}+\frac{a_{2}+b_{2}}{2} u+\frac{c_{2}+d_{2}}{2} v+O(2) .
\end{aligned}
$$


Thus $H_{\alpha}=H_{1} N_{1}+H_{2} N_{2}$ can be written as follows:

$$
\begin{aligned}
H_{\alpha}= & \left(-\left(r_{1} t_{1}+r_{1}^{2}+r_{2} t_{2}+r_{2}^{2}\right) \frac{u}{2}-\left(s_{1} t_{1}+s_{1} r_{1}+s_{2} t_{2}+r_{2} s_{2}\right) \frac{v}{2}+O(2),\right. \\
- & \left(s_{1} t_{1}+s_{1} r_{1}+s_{2} t_{2}+r_{2} s_{2}\right) \frac{u}{2}-\left(t_{1}^{2}+r_{1} t_{1}+t_{2}^{2}+r_{2} t_{2}\right) \frac{v}{2}+O(2), \\
& \frac{t_{1}+r_{1}}{2}+\left(a_{1}+b_{1}\right) \frac{u}{2}+\left(c_{1}+d_{1}\right) \frac{v}{2}+O(2), \\
& \left.\frac{t_{2}+r_{2}}{2}+\left(a_{2}+b_{2}\right) \frac{u}{2}+\left(c_{2}+d_{2}\right) \frac{v}{2}+O(2)\right)
\end{aligned}
$$

Thus, in the chart $(u, v)$, a normal singularity located at 0 is characterized by $\left(r_{1}+t_{1}=0, r_{2}+t_{2}=0\right)$. Also the differential equation 11 of principal mean curvature lines in the chart $(u, v)$ around such a normal singularity is given by:

$$
\begin{gathered}
\left\{-\left[s_{1}\left(a_{1}+b_{1}\right)+s_{2}\left(a_{2}+b_{2}\right)\right] \frac{u}{2}-\left[s_{1}\left(c_{1}+d_{1}\right)+s_{2}\left(c_{2}+d_{2}\right)\right] \frac{v}{2}+O_{1}\right\} d v^{2} \\
-\left[\left(r_{1}\left(a_{1}+b_{1}\right)+r_{2}\left(a_{2}+b_{2}\right)\right) u+\left(r_{1}\left(c_{1}+d_{1}\right)+r_{2}\left(c_{2}+d_{2}\right)\right) v+O_{2}\right] d u d v+ \\
\left\{\left[s_{1}\left(a_{1}+b_{1}\right)+s_{2}\left(a_{2}+b_{2}\right)\right] \frac{u}{2}+\left[s_{1}\left(c_{1}+d_{1}\right)+s_{2}\left(c_{2}+d_{2}\right)\right] \frac{v}{2}+O_{3}\right\} d u^{2}=0 \\
:=-\left(\bar{d} u+\bar{b} v+O_{1}\right) d v^{2}+\left[\bar{a} u+\bar{c} v+O_{2}\right] d u d v+\left(\bar{d} u+\bar{b} v+O_{3}\right) d u^{2}=0
\end{gathered}
$$

where, $O_{i}=O\left(u^{2}+v^{2}\right)$.

Remark 1. After an appropriate rotation in the frame $(u, v)$, it can be assumed that $\bar{d}=s_{1}\left(a_{1}+b_{1}\right)+s_{2}\left(a_{2}+b_{2}\right)=0$. In fact, the equation 8 in the coordinates $\left(u_{1}, v_{1}\right)$, where $u=\cos \omega u_{1}+\sin \omega v_{1}, \quad v=-\sin \omega u_{1}+$ $\cos \omega v_{1}$, is given by:

$-\left[\bar{d}_{1} u_{1}+\bar{b}_{1} v_{1}\right] d v_{1}^{2}+\left[\bar{a}_{1} u_{1}+\bar{c}_{1} v_{1}\right] d u_{1} d v_{1}+\left[\bar{d}_{1} u_{1}+\bar{b}_{1} v_{1}\right] d u_{1}^{2}+O(2)=0$ where,

$\bar{d}_{1}=\cos ^{3} \omega\left[\bar{b} \tan ^{3} \omega+(\bar{c}-\bar{d}) \tan ^{2} \omega-(\bar{a}+\bar{b}) \tan \omega+\bar{d}\right]$

Solving the cubic equation $\bar{d}_{1}(\tan \omega)=0$ the assertion follows.

A normal singularity is called Darbouxian if

a) $H_{\alpha}$ is transversal to the zero section of the normal bundle:

$\bar{a} \bar{b}-\bar{c} \bar{d}=\bar{a}_{1} \bar{b}_{1}-\bar{c}_{1} \bar{d}_{1}=\frac{1}{2} b_{1}\left(c_{1}+d_{1}\right) s_{1} r_{1}+\frac{1}{2} b_{2}\left(c_{2}+d_{2}\right) s_{2} r_{2}+\frac{1}{2}\left[\left(a_{1}+\right.\right.$ $\left.\left.b_{1}\right)\left(c_{2}+d_{2}\right)-a_{2}\left(c_{1}+d_{1}\right)\right] s_{1} r_{2}+\frac{1}{2}\left[\left(a_{2}+b_{2}\right)\left(c_{1}+d_{1}\right)-a_{1}\left(c_{2}+d_{2}\right)\right] s_{2} r_{1} \neq 0$ and

b) one of the following condition holds:

$\left.D_{1}\right) \bar{d}_{1}=0, \bar{c}_{1}^{2}+4 \bar{b}_{1}\left(\bar{a}_{1}+\bar{b}_{1}\right)<0$,

$\left.D_{2}\right) \bar{d}_{1}=0, \bar{c}_{1}^{2}+4 \bar{b}_{1}\left(\bar{a}_{1}+\bar{b}_{1}\right)>0,-1 \neq \bar{a}_{1} / \bar{b}_{1}<0$, 
$\left.D_{3}\right) \bar{d}_{1}=0, \bar{a}_{1} / \bar{b}_{1}>0$.

Remark 2. It can be shown that the conditions $D_{i}$ above are independent of the rotation performed to have $\bar{d}_{1}(\tan w)=0$ in Remark [1.

The differential equation 1 of mean curvature lines near the umbilic singularity 0 characterized by $e_{H} G-g_{H} E=t_{1}^{2}+t_{2}^{2}-r_{1}^{2}-r_{2}^{2}=0$ and $f_{H} G-g_{H} F=s_{1}\left(t_{1}+r_{1}\right)+s_{2}\left(t_{2}+r_{2}\right)=0$, is given by:

$$
\begin{aligned}
& -\left\{\left[2 r_{1} d_{1}+s_{1}\left(a_{2}+b_{2}\right)\right] \frac{u}{2}+\left[2 r_{1} b_{1}+s_{1}\left(c_{2}+d_{2}\right)\right] \frac{v}{2}+O_{1}(2)\right\} d v^{2} \\
& +\left\{\left[r_{1}\left(b_{1}-a_{1}\right)-r_{2}\left(a_{2}+b_{2}\right)\right] u+\left[r_{1}\left(c_{1}-d_{1}\right)-r_{2}\left(c_{2}+d_{2}\right)\right] v+O_{2}(2)\right\} d u d v \\
& +\left\{\left[2 r_{1} d_{1}+s_{1}\left(a_{2}+b_{2}\right)\right] \frac{u}{2}+\left[2 r_{1} b_{1}+s_{1}\left(c_{2}+d_{2}\right)\right] \frac{v}{2}+O_{3}(2)\right\} d u^{2}=0 \\
& :=-(\tilde{d} u+\tilde{b} v) d v^{2}+[\tilde{a} u+\tilde{c} v] d u d v+(\tilde{d} u+\tilde{b} v) d u^{2}+O(2)=0
\end{aligned}
$$

Remark 3. As in the normal singularity case by an appropriate rotation in the plane $(u, v)$ it can be assumed that $\tilde{d}=2 r_{1} d_{1}+s_{1}\left(a_{2}+b_{2}\right)=0$.

A umbilic singularity is called Darbouxian if

a) $\mathcal{W}_{\alpha}$, regarded as a section is transversal to the line bundle of diagonal operators; in terms of the coefficients defined in 10, this transversality condition writes: $\tilde{a} \tilde{b}-\tilde{c} \tilde{d}=\left[b_{1}\left(b_{1}-a_{1}\right)+d_{1}\left(d_{1}-c_{1}\right)\right] r_{1}^{2}+\left[d_{1}\left(c_{2}+d_{2}\right)-\right.$ $\left.b_{1}\left(a_{2}+b_{2}\right)\right] r_{1} r_{2}+\frac{1}{2}\left[\left(a_{2}+b_{2}\right)\left(d_{1}-c_{1}\right)+\left(b_{1}-a_{1}\right)\left(c_{2}+d_{2}\right)\right] r_{1} s_{2} \neq 0$. and b) one of the following condition, expressed assuming the simplification in Remark 3, holds:

$\left.D_{1}\right) \tilde{d}=0, \quad \tilde{c}^{2}+4 \tilde{b}(\tilde{a}+\tilde{b})<0$,

$\left.D_{2}\right) \tilde{d}=0, \quad \tilde{c}^{2}+4 \tilde{b}(\tilde{a}+\tilde{b})>0, ;-1 \neq \tilde{a} / \tilde{b}<0$,

$\left.D_{3}\right) \tilde{d}=0, \quad \tilde{a} / \tilde{b}>0$.

The local behavior of the foliations $\mathcal{F}_{m, \alpha}$ and $\mathcal{F}_{M, \alpha}$ near singularities are as shown in the Fig. 11.

The conditions $D_{i}$ given above are similar to those obtained by Gutierrez and Sotomayor [5] to characterize Darbouxian umbilic points of surfaces of $\mathbb{R}^{3}$. We have the following correspondences with the $(a, b, c)$ notation of [5] and [7]: $\bar{b}_{1}=b, \bar{c}_{1}=c, \bar{a}_{1}=b-a$, for normal singularities, and $\tilde{b}_{1}=b, \tilde{c}_{1}=c, \tilde{a}_{1}=b-a$, for umbilic singularities.

The proof of the local configurations for both cases is therefore the same as in [5] and [7], applied to the equilibria of the Lie-Cartan Vector Field [2, with $J_{\alpha}$ as in left hand members of the last equations in 8 and 10. 


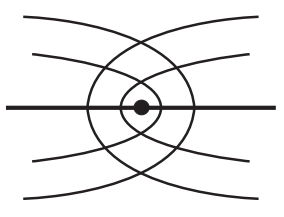

$D_{1}$

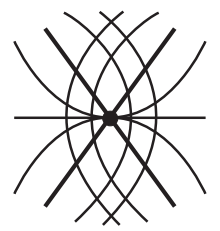

$\mathrm{D}_{2}$

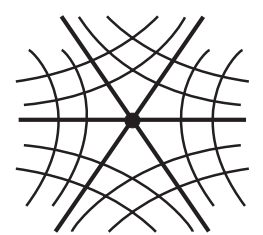

$\mathrm{D}_{3}$

Figure 1. Normal and Umbilic Darbouxian Singularities and their Separatrices.

\section{Hyperbolic Cycles}

Let $\alpha \in \mathcal{I}^{r, s}$ and suppose that $c$ is a regular arc length parametrized curve in $\mathbb{M}^{2} \backslash \mathcal{S}_{\alpha}$. Call $t=c^{\prime}$ the tangent vector field of $c$ and let $T$ be the unit vector field along $c$ such that the tangent frame $\{t, T\}$ is positive.

The equations of Darboux for the frame $\left\{t, T, N:=N_{\alpha}, B:=B_{\alpha}\right\}$ along $c$ are given by:

$$
\begin{aligned}
t^{\prime} & =k_{g} T+k N+k_{B} B, \quad T^{\prime}=-k_{g} t-\tau_{N} N-\tau B, \\
N^{\prime} & =-k t+\tau_{N} T+\tau_{B} B, \quad B^{\prime}=-k_{B} t+\tau T-\tau_{B} N .
\end{aligned}
$$

Lemma 1. Let $c$ be a minimal principal mean cycle of length $L$ of an immersed surface $\mathbb{M}^{2}$ in $\mathbb{R}^{4}$.

Then the expression

$$
\begin{aligned}
\alpha(u, v) & =c(u)+v T(u)+\left[K(u) \frac{v^{2}}{2}+a(u) \frac{v^{3}}{6}+v^{3} A(u, v)\right] N(u) \\
& +\left[\bar{K}(u) \frac{v^{2}}{2}+b(u) \frac{v^{3}}{6}+v^{3} \bar{A}(u, v)\right] B(u),
\end{aligned}
$$

where $A(u, 0)=\bar{A}(u, 0)=0$, defines a $L$ - periodic chart in a neighborhood of $c$.

Proof. See [3] and [5].

With the notation in equations 11 and 12, follows that $c$ is a minimal principal mean cycle if and only if the following holds along it,

$$
\tau_{N} \equiv 0, \quad k_{B}+\bar{K} \equiv 0, \quad K-k>0 .
$$


Lemma 2. Let $c$ be a principal mean curvature cycle and consider a coordinate chart $(u, v)$ as in Lemma 1, Then the first derivative of the Poincaré map $\pi$ of the principal cycle $c$ has the form

$$
\ln \pi^{\prime}(0)=\int_{0}^{L} \frac{-\left[E f_{H}-F e_{H}\right]_{v}}{E g_{H}-G e_{H}} d u
$$

where $L$ is the length of the principal cycle, $E, F, G$ are the coefficients of the first fundamental form and $e_{H}, f_{H}, g_{H}$ are the coefficients of the second fundamental form with respect to the normal vector field $H_{\alpha}$ given in equation [1, calculated relative to the chart $(u, v)$ defined by 12.

Proof. The differential equation of principal mean curvature lines is given in equation [1]

As $F(u, 0)=f_{H}(u, 0)=0$, the result follows by differentiating the equation above with respect to the initial condition $v_{0}$ - thus getting the linear variational equation-. Recall that $\pi\left(v_{0}\right)=v\left(L, v_{0}\right)$ where $v\left(u, v_{0}\right)$ is the solution of this equation with initial condition $v\left(0, v_{0}\right)=v_{0}$. The expression for $\pi^{\prime}(0)$ in equation 14 follows from the integration of the linear variational equation.

The calculation that follows culminates in an expression of the integral in equation 14 in terms of the functions of the arc length $u$ defined in equations 11, 12 and 13, leading to the integral in Proposition 1.

$$
\begin{aligned}
\alpha_{u}(u, v) & =\left(1-k_{g} v-k_{B} A_{2}\right) t+\left(\tau_{N} A_{1}+\tau A_{2}\right) T \\
& +\left(\frac{\partial A_{1}}{\partial u}-\tau_{N} v-\tau_{B} A_{2}\right) N+\left(\frac{\partial A_{2}}{\partial u}-\tau v-\tau_{B} A_{1}\right) B \\
\alpha_{v}(u, v) & =T+\frac{\partial A_{1}}{\partial v} N+\frac{\partial A_{2}}{\partial v} B \\
A_{1}(u, v) & =K(u) \frac{v^{2}}{2}+a(u) \frac{v^{3}}{6}+v^{3} A(u, v) \\
A_{2}(u, v) & =\bar{K}(u) \frac{v^{2}}{2}+b(u) \frac{v^{3}}{6}+v^{3} \bar{A}(u, v) .
\end{aligned}
$$

Write $\alpha_{u}=x_{1} t+x_{2} T+x_{3} N+x_{4} B$ and $\alpha_{v}=T+y_{1} N+y_{2} B$. Let $\bar{N}_{1}=\left(y_{1} x_{2}-x_{3}\right) t-x_{1} y_{1} T+x_{1} N+0 B$ and $\bar{N}_{2}=\alpha_{u} \wedge \alpha_{v} \wedge \bar{N}_{1}$. 
Then it follows that $<\bar{N}_{i}, \alpha_{u}>=<\bar{N}_{i}, \alpha_{v}>=<\bar{N}_{1}, \bar{N}_{2}>=0$. Direct calculations show that

$$
\begin{aligned}
\bar{N}_{2} & =\left(x_{1} x_{2} y_{2}-x_{1} x_{4}+x_{1} x_{3} y_{1} y_{2}-x_{1} x_{4} y_{1}^{2}\right) t \\
& +\left(-x_{1}^{2} y_{2}+x_{2} x_{3} y_{1} y_{2}-x_{2} x_{4} y_{1}^{2}-x_{3}^{2} y_{2}+x_{3} x_{4} y_{1}\right) T \\
& +\left(-x_{1}^{2} y_{1} y_{2}-x_{2}^{2} y_{1} y_{2}+x_{2} x_{4} y_{1}+x_{2} x_{3} y_{2}-x_{3} x_{4}\right) N \\
& +\left(x_{1}^{2}+x_{1}^{2} y_{1}^{2}+x_{2}^{2} y_{1}^{2}-2 x_{2} x_{3} y_{1}+x_{3}^{2}\right) B .
\end{aligned}
$$

Let $N_{1}(u, v)=\bar{N}_{1} /\left|\bar{N}_{1}\right|$ and $N_{2}(u, v)=\bar{N}_{2} /\left|\bar{N}_{2}\right|$ be orthonormal vector fields.

Straightforward calculations lead to:

$$
\begin{aligned}
& N_{1}(u, v)=\left[\tau_{N} v+O(2)\right] t+[-K v+O(2)] T+[1+O(2)] N \\
& N_{2}(u, v)=[\tau v+O(2)] t+[-\bar{K} v+O(2)] T+[O(2)] N+[1+O(2)] B
\end{aligned}
$$

From equation [15] it follows that

$$
\begin{aligned}
E(u, 0) & =G(u, 0)=1, F(u, 0)=0 \\
E_{v}(u, 0) & =-2 k_{g}(u), F_{v}(u, 0)=G_{v}(u, 0)=0 .
\end{aligned}
$$

Also from equations [1], 12 and [17 it follows that

$$
\begin{aligned}
e_{1}(u, 0) & =k(u), \quad f_{1}(u, 0)=-\tau_{N}, \quad g_{1}(u, 0)=K(u) \\
\left(e_{1}\right)_{v}(u, 0) & =-k_{g}(k+K)+\tau \tau_{B}-\tau_{N}^{\prime} \\
\left(f_{1}\right)_{v}(u, 0) & =K^{\prime}-\bar{K} \tau_{B}-k_{g} \tau_{N} \\
\left(g_{1}\right)_{v}(u, 0) & =a(u) .
\end{aligned}
$$

Here $e_{1}(u, v)=<\alpha_{u u}, N_{1}(u, v)>, f_{1}(u, v)=<\alpha_{u v}, N_{1}(u, v)>$ and $g_{1}(u, v)=<\alpha_{v v}, N_{1}(u, v)>$.

From $e_{2}(u, v)=<\alpha_{u u}, N_{2}(u, v)>, f_{2}(u, v)=<\alpha_{u v}, N_{2}(u, v)>$ and $g_{2}(u, v)=<\alpha_{v v}, N_{2}(u, v)>$, it follows that:

$$
\begin{aligned}
e_{2}(u, 0) & =k_{B}(u), \quad f_{2}(u, 0)=-\tau(u), \quad g_{2}(u, 0)=\bar{K}(u) \\
\left(e_{2}\right)_{v}(u, 0) & =-k_{g}\left(k_{B}+\bar{K}\right)-\tau_{N} \tau_{B}-\tau^{\prime} \\
\left(f_{2}\right)_{v}(u, 0) & =\bar{K}^{\prime}+K \tau_{B}-k_{g} \tau \\
\left(g_{2}\right)_{v}(u, 0) & =b(u) .
\end{aligned}
$$

Define

$$
\tilde{H}_{i}=\frac{E g_{i}-2 f_{i} F+e_{i} G}{2\left(E G-F^{2}\right)}, \quad i=1,2
$$


Accordingly, the mean curvature vector writes $H_{\alpha}(u, v)=\tilde{H}_{1} N_{1}(u, v)+$ $\tilde{H}_{2} N_{2}(u, v)$.

From equations [19, 20] and 21] it follows that:

$$
\begin{aligned}
\tilde{H}_{1}(u, 0) & =\frac{k+K}{2}, \quad \tilde{H}_{2}(u, 0)=\frac{k_{B}+\bar{K}}{2}=0 \\
\left(\tilde{H}_{1}\right)_{v} & =a(u)-k_{g}(K-k)+\tau \tau_{B}-\tau_{N}^{\prime} \\
2\left(\tilde{H}_{2}\right)_{v} & =b(u)-2 k_{g} \bar{K}-\tau_{N} \tau_{B}-\tau^{\prime}
\end{aligned}
$$

Therefore it follows from equations [17 and 22 that the functions $e_{H}(u, v)=<\alpha_{u u}, H_{\alpha}(u, v)>, f_{H}(u, v)=<\alpha_{u v}, H_{\alpha}(u, v)>$ and $g_{H}(u, v)=<$ $\alpha_{v v}, H_{\alpha}(u, v)>$ evaluated at $v=0$ give:

$$
\begin{aligned}
e_{H}(u, 0) & =k \tilde{H}_{1}(u, 0), \quad f_{H}(u, 0)=-\tau_{N} \tilde{H}_{1}=0, g_{H}(u, 0)=K \tilde{H}_{1}(u, 0) \\
\left(f_{H}\right)_{v}(u, 0) & =\tilde{H}_{1}(u, 0)\left(K^{\prime}+\tau_{B} \bar{K}\right)+\left(\tilde{H}_{2}\right)_{v} \tau
\end{aligned}
$$

Proposition 1. The first derivative of the Poincaré map of a minimal principal cycle is given by

$$
\ln \pi^{\prime}(0)=-\int_{0}^{L} \frac{k^{\prime}}{K-k} d u+\int_{0}^{L} \frac{k_{B} \tau_{B}}{K-k} d u-\int_{0}^{L} \frac{\left(\tilde{H}_{2}\right)_{v}(u, 0) \tau}{\tilde{H}_{1}(u, 0)(K-k)} d u
$$

Proof. It follows directly from lemma 2 equations 22 and 23 and integration by parts.

Remark 4. In the last integral, the expressions involving $\tilde{H}_{1}$ and $\left(\tilde{H}_{2}\right)_{v}$ can be further simplified using the equations in 22. Notice that this introduces $b(u)$ which however can itself be expressed in terms of the three dimensional torsion of the curve $v \rightarrow \alpha(u, v)$ in the 3-space generated by $\{T(u), N(u), B(u)\}$.

The next proposition shows how to deform an immersion making hyperbolic a cycle, under mild conditions.

Proposition 2. Consider the one parameter family of immersions:

$$
\alpha_{\epsilon}(u, v)=\alpha(u, v)+\epsilon \delta(u) m(v) \frac{v^{3}}{6} B(u)
$$

where $m(v)=1$ in neighborhood of $v=0$, with small support and $\delta>0$.

If $\tau \neq 0$, then $c$ is a hyperbolic principal cycle for all immersions $\alpha_{\epsilon}, \epsilon>0$ small. 
Proof. Along $c$ the deformation $\alpha_{\epsilon}$ given by equation [25] has the same second order jet as that of $\alpha$. It follows that $c$ is also an arc length parametrized minimal principal mean curvature cycle for $\alpha_{\epsilon}$. In the integral expression 24 for the derivative of the Poincaré map it follows that $\left(\tilde{H}_{2}\right)_{v}(u, 0, \epsilon)=\left(\tilde{H}_{2}\right)_{v}(u, 0)+\epsilon \delta(u)$ while all the other functions involved are independent on $\epsilon$. Therefore, after a direct calculation, it

follows that $\left.\frac{\partial}{\partial \epsilon}\left(\ln \pi_{\epsilon}^{\prime}\right)(0)\right|_{\epsilon=0}=-\int_{0}^{L} \frac{\tau \delta}{\tilde{H}_{1}(K-k)} d u$, which is positive taking $\delta=-\tau \tilde{H}_{1}$.

\section{Outline of the Proof of Theorem 1}

Once the hypotheses on $\alpha$ are expressed in the Projective Tangent bundle of $\mathbb{M}^{2}$ and identified with those for the quadratic equation 1 which, in turn, amount to the hyperbolicity of equilibria and periodic orbits of the Lie-Cartan Line Field, locally expressed by $X_{\alpha}$ in 2 , the similarity with the case of principal line fields dealt with in [5] and [7] becomes evident. In fact, the construction and continuation to a small neighborhood $\mathcal{V}(\alpha)$ of $\alpha$ of the canonical regions follow also from the openness and unique continuation, for $\beta$ near $\alpha$, of the singularities (and their separatrices and parabolic sectors) and of cycles (and their local invariant manifolds), due to the hyperbolicity of these elements in the field $X_{\alpha}$. This leads to the openness of $\Sigma^{r, s}$ and gives the uniqueness of the correspondence between singularities, normal and umbilic, separatrices, cycles for both minimal and maximal foliations involved and their intersections for $\mathcal{P}_{\alpha}$ and $\mathcal{P}_{\beta}$. The extension of this correspondence to define a topological equivalence homeomorphism $h_{\beta}$, is carried out as in the case of principal configurations [5].

\section{Concluding Remarks and Related Problems}

The study of the bending of a surface immersed in $\mathbb{R}^{4}$, focusing the stability properties of the integral foliations defined by geometric properties related to certain normal line fields, has a rich background. The approach and pertinent results presented here should be considered in the perspective of previous achievements. A concise discussion follows.

The axial configuration of Garcia and Sotomayor [3, for which the normal line fields are those of the principal axes of the ellipse of curvature, may be richest of all them. In fact, it leads to fields of tangent crosses rather than to tangent line fields. This theory, when restricted to a surface immersed in $\mathbb{R}^{3}$, reduces to both the standard principal and that of the arithmetic mean [4] configurations. 
Garcia and Sotomayor 2] have studied principal cycles of immersions of surfaces in a three dimensional Riemannian manifold. The expression of the derivative of the return map should be compared with that of equation 24 .

By taking the normal line field to be an arbitrary unit vector field, $\nu$, Garcia and Sánchez [1] have obtained an integral expression for the first derivative of the return map associated to a principal cycle.

Mello [11] has considered the tangent line fields defined by the property of having their normal curvature vector parallel to $H_{\alpha}$. The approach of the present paper is in between this and the previous one.

The consideration of other geometric normal vector fields such as $\nu=B_{\alpha}$, the bi-normal, instead of $H_{\alpha}$ in the present paper, may be also of interest.

The $C^{2}$ density of the limit set triviality condition seems to be most difficult problem left open here; see Theorem 1. This problem is also present and, as far as we know, still open for the previous approaches mentioned above.

Other direction of research, though not directly related to stability, emerges with the evaluation of the Index of an isolated singularity of $\mathcal{P}_{\alpha}$. This is related to the upper bound 1 for the umbilic index on surfaces in $\mathbb{R}^{3}$, connected to deep problems around the Carathéodory Conjecture. See Smyth and Xavier [12] and Ivanov [9].

Gutierrez and Sánchez [8] have shown that this bound does not hold for the $\nu$ approach. The case of $\nu=H_{\alpha}$ presented here contrasts with the flexibility in the case of arbitrary $\nu$. The question of the upper bound of the index of a singularity seems more difficult to analyze in the present case. 


\section{REFERENCES}

[1] R. Garcia and F. Sánchez B., J. Dyn. and Control Systems, 08, 153, (2002).

[2] R. Garcia and J. Sotomayor, Pitman Res. Notes, 285, 255, (1993).

[3] R. Garcia and J. Sotomayor, Diff. Geo. and its Applications, 12, 253, (2000).

[4] R. Garcia and J. Sotomayor, Publ. Matemátiques, 45, 431, (2001).

[5] C. Gutierrez and J. Sotomayor, Asterisque, 98-99, 191 (1982).

[6] C. Gutierrez and J. Sotomayor, Lect. Notes in Math. 1007, 332, (1983).

[7] C. Gutierrez and J. Sotomayor, Lines of Curvature and Umbilical Points on Surfaces, IMPA, (1991).

[8] C. Gutierrez and F. Sánchez B., J. Dyn. and Control Systems, 04, 127, (1998).

[9] V. V. Ivanov, Siberian Math. Journal, 43, 251, (2002).

[10] J. A. Little, Ann. di Mat. Pura App., 83, 261, (1969).

[11] L. F. Mello, Publ. Matemátiques, 47, 415, (2003).

[12] B. Smyth and F. Xavier, Bull. London Math. Soc. 24, 176 , (1992).

Instituto de Matemática e Estatística

Universidade Federal de Goiás

Caixa Postal 131

74001-970 Goiânia, GO, Brasil

E-mail: ragarcia@mat.ufg.br

Instituto de Ciências

Universidade Federal de Itajubá

37500-903 Itajubá, MG, Brasil

E-mail: lfmelo@unifei.edu.br

Instituto de Matemática e Estatística

Universidade de São Paulo

Rua do Matão 1010, Cidade Universitária

05508-090 São Paulo, SP, Brasil

E-mail: sotp@ime.usp.br 